\title{
Molecular Characterization and Detection of a Genetically Distinct Tomato Chlorosis Virus Strain in Taiwan
}

Ya-Chi Kang, Department of Plant Pathology, National Chung Hsing University, Taichung 40227, Taiwan; Yun-Chi Wang and Chun-Ming Hsia, Department of Biotechnology, Asia University, Wufeng, Taichung 41354, Taiwan; Wen-Shi Tsai, Department of Plant Medicine, National Chiayi University, Chiayi 60004, Taiwan; Li-Hsin Huang, Taiwan Agricultural Chemicals and Toxic Substances Research Institute, Wufeng, Taichung 41358, Taiwan; Shyi-Dong Yeh, ${ }^{\dagger}$ Department of Plant Pathology, National Chung Hsing University, Taichung 40227, Taiwan; and Tsung-Chi Chen, ${ }^{\dagger}$ Department of Biotechnology, Asia University, Wufeng, Taichung 41354, Taiwan, and Department of Medical Research, China Medical University Hospital, China Medical University, Taichung 40402, Taiwan

\begin{abstract}
The whitefly-transmitted tomato chlorosis virus (ToCV) belonging to the genus Crinivirus (family Closteroviridae) affects tomato production worldwide. ToCV was first recorded in Taiwan in 1998 affecting tomato production. In this study, a local virus isolate XS was obtained, after serial whitefly transmissions from a diseased tomato plant displaying general chlorosis were collected in central Taiwan. The whole genome sequence of XS was determined from cDNA fragments amplified by reverse transcription (RT)-PCR, first using the degenerate primers for viruses of Closteroviridae and followed by degenerate and specific primers designed on available sequences of the ToCV isolates. The nucleotide (nt) sequences of RNA-1 and RNA-2 of the XS shared low identities of 77.8 to $78 \%$ and 78 to $78.1 \%$, respectively, with genome segments of other ToCV isolates. Nevertheless,

the viral RNA-dependent RNA polymerase (RdRp), heat shock protein 70 homolog (Hsp70h), and major capsid protein (CP) shared 88.3 to $96.2 \%$ amino acid (aa) identities with other ToCV isolates, indicating that $\mathrm{XS}$ is a new strain of this virus. Phylogenetic analyses of these three proteins indicated that all ToCV isolates from different counties outside Taiwan are closely related and clustered in the same clade, whereas the XS isolate is distinct and forms a unique branch. A one tube RT-PCR assay using primers designed from the genomic sequence of the XS was able to detect the ToCV-XS in infected tomato plants and in individual whiteflies. A field survey during 2013 to 2016 revealed a high ToCV-XS prevalence of $60.5 \%$ in 172 tested tomato samples, demonstrating that ToCV-XS is becoming an emerging threat for tomato production in Taiwan.
\end{abstract}

The family Closteroviridae comprises four genera, Ampelovirus, Closterovirus, Crinivirus, and Velarivirus. Members of Ampelovirus, Closterovirus, and Crinivirus are transmitted by mealybugs, aphids, and whiteflies, respectively, while the vector of velariviruses remains unknown (Al Rwahnih et al. 2012; Jelkmann et al. 2012; Martelli et al. 2012). Most viruses within the family Closteroviridae are phloem-limited and difficult to be mechanically introduced to host plants (Martelli et al. 2011). Unlike members of the genera Ampelovirus, Closterovirus, and Velarivirus, which contain a monopartite genome and nonenveloped filamentous particles 1,350 to $2,000 \mathrm{~nm}$ in length, the criniviruses possess a bipartite genome encapsidated in filamentous particles of 750 to $900 \mathrm{~nm}$. The $3^{\prime}$ ends of criniviral RNA segments are not polyadenylated and the $5^{\prime}$ ends are likely to be capped (Martelli et al. 2011).

The numbers of open reading frames (ORFs) within RNA-1 and RNA2 vary among different criniviruses. Two ORFs, ORF1a and ORF1b, associated with virus replication are encoded by RNA-1 (Martelli et al. 2011). ORF1a encodes a protein containing domains of papain-like protease (P-Pro), methyl transferase (MT), and helicase (HEL), and ORF1b encodes an RNA-dependent RNA polymerase (RdRp) by ribosomal frameshifting. The ORF numbers downstream ORF1b are virus-specific, ranging from zero to three (Kataya et al. 2009). Most criniviral RNA-1

${ }^{\dagger}$ Corresponding authors: Shyi-Dong Yeh, E-mail: sdyeh@nchu.edu.tw; and Tsung-Chi Chen, E-mail: kikichenwolf@hotmail.com

We thank the funding supports from the Bureau of Animal and Plant Health Inspection and Quarantine, Council of Agriculture, Executive Yuan [101AS10.1.2-BQ-B2 and 102AS-10.1.2-BQ-B1] and the Ministry of Science and Technology, R.O.C. [MOST103-2313-B-468-001 and MOST104-2313-B468-001] for this study.

*The $\boldsymbol{e}$-Xtra logo stands for "electronic extra" and indicates that two supplementary tables and one supplementary figure are published online.

Accepted for publication 14 October 2017.

() 2018 The American Phytopathological Society segments encode a protein of 22 to $25 \mathrm{kDa}$ that functions as an RNA silencing suppressor (RSS) (Kataya et al. 2009; Landeo-Ríos et al. 2016). Criniviral RNA-2 contains a "hallmark gene array" consisting of a heat shock protein 70 homolog (Hsp70h), a protein around 59 to $60 \mathrm{kDa}$ (P59 or P60), a major capsid protein (CP), and a minor capsid protein (CPm) (Karasev 2000) that are components of criniviral virion (Tian et al. 1999). The criteria for classification of criniviruses include particle size, vector specificity, host range, serological relationship, genomic organization, cytopathological features, and a threshold of $75 \%$ amino acid (aa) identity of RdRp, Hsp70h, and CP (Martelli et al. 2011).

Tomato chlorosis virus (ToCV), a member of the genus Crinivirus, can be transmitted by several whitefly species, such as the species New World, Middle East-Asia Minor 1, and Mediterranean (formerly biotypes A, B, and Q, respectively) of the Bemisia tabaci (Gennadius) complex Trialeurodes vaporariorum (Westwood), in a semipersistent manner (Navas-Castillo et al. 2000; Wintermantel and Wisler 2006; Wisler et al. 1998). ToCV is one of the most important tomatoinfecting viruses, causing severe economic losses by reducing fruit yield and delaying ripening (Wintermantel and Wisler 2006). The outbreaks of the ToCV are frequently associated with high populations of whiteflies. ToCV has become an emerging disease of tomato crops in more than 20 countries of Africa, Asia, America, and Europe (Al Saleh et al. 2014; Dovas et al. 2002; Fiallo-Olivé et al. 2011; Hirota et al. 2010; Moodley et al. 2016; Navas-Castillo et al. 2011; Zhao et al. 2013), but the genetic diversity of the ToCV has not been studied so far.

ToCV was first found in tomato and zinnia plants in the Shanhua area of southern Taiwan in 1998 (Tsai et al. 2004). The virus has become a serious problem for tomato production in central Taiwan since 2011. In this study, the causal virus of the tomato chlorosis disease was isolated through serial transmissions of the whitefly B. tabaci B biotype from a diseased sample collected from Xinshe District of Taichung City in central Taiwan, referred to as XS, and characterized. We demonstrated here that the XS represents a new strain of ToCV, phylogenetically divergent from other isolates described up to now.

\section{Materials and Methods}

Virus identification and isolation. The diseased tomato samples showing interveinal chlorosis on leaves (Fig. 1A and B, left) were 
collected from Xinshe District, Taichung City, in December 2011. The total RNAs of the field tomato samples were extracted from the leaf tissues using the Plant Total RNA Miniprep Purification kit (GMbiolab, Taichung, Taiwan) according to the manufacturer's instructions and used as templates. A previously described nested reverse transcription (RT)-PCR method using the degenerate primer set, dHSP up1/dHSP up1G/dHSP do2/dHSP do2C, for RT-PCR and another set for nested PCR was performed to examine the possible presence of viruses in the family Closteroviridae (Dovas and Katis 2003). The final PCR products were sequenced and analyzed by the basic local alignment search tool (BLAST) of the National Center for Biotechnology Information (NCBI, https://www.ncbi.nlm.nih. gov/) to identify virus species.

The procedure of whitefly transmission was conducted following a previous report (Navas-Castillo et al. 2000) with modifications to obtain the possible causal virus of a tomato chlorosis sample. Briefly, the symptomatic tomato leaves were cut into small pieces $(1.5 \times$ $1.5 \mathrm{~cm}^{2}$ ) and placed in 50-ml centrifuge tubes, and then 20 to 30 individuals of virus-free whitefly $B$. tabaci B biotype were transferred into the centrifuge tubes for a 48 -h acquisition access period. Thereafter, individual whiteflies were transferred into an insect rearing cage $(30 \mathrm{~cm} \times 30 \mathrm{~cm} \times 30 \mathrm{~cm})$ with $150 \times 150$ mesh nylon nets containing 10-day-old seedlings of healthy tomato cv. Bucolic1105 (Bucolic seeds Co., Ltd., Taichung, Taiwan) in a greenhouse at $28 \pm 0.5^{\circ} \mathrm{C}$, for an inoculation access period of $72 \mathrm{~h}$. The plants were grown for 4 weeks in an isolated and temperature-controlled greenhouse (23 to $28^{\circ} \mathrm{C}$ ) for observation of symptom development, and this process was serially repeated three times to get a putative pure virus culture. A virus isolate thus obtained was denoted XS and maintained in the plants of tomato cv. Bucolic1105 by whitefly transmission for further study.

Sequencing of viral genome. The DNA fragments amplified from the diseased tomato samples by the nested RT-PCR method shared the highest homology ( $82 \%$ nt identity) with the Hsp70h sequence of the ToCV AT80/99 isolate (accession no. DQ136146) from which the whole genome sequence is available. Thus, the genomic sequence of ToCV-AT80/99 was used as the reference for designing primers for the genome sequencing of XS. Degenerate primers were used to amplify the regions in which the ToCV-AT80/99-specific primers failed to amplify. Degenerate primers were designed from the multiple alignments of the genomic sequences of different ToCV isolates (Table 1), conducted by the ClustalW program of Biology Workbench, San
Diego Supercomputer Center (SDSC) (http://workbench.sdsc.edu/). The XS-specific primers were designed from the determined sequences to amplify longer overlapping fragments for verifying the fidelity of the genome sequence. All sequences of primers used for genome sequencing of XS are shown in Supplementary Table S1.

The total RNA extracted from the XS-infected tomato plant was used as the template for further characterization. RT was performed using $1 \mu \mathrm{g}$ of total RNA mixed with $200 \mathrm{nM}$ individual primers and $20 \mathrm{U}$ of high fidelity SuperScript III reverse transcription (Invitrogen, Carlsbad, CA). The cDNA synthesis reaction was inactivated by heating at $75^{\circ} \mathrm{C}$ for $15 \mathrm{~min}$. Subsequently, $2 \mu \mathrm{l}$ of cDNA was heated at $94^{\circ} \mathrm{C}$ for $2 \mathrm{~min}$, placed on ice for $1 \mathrm{~min}$, and mixed with $2.5 \mathrm{U}$ Ex Taq DNA polymerase (Takara, Shiga, Japan), $200 \mathrm{nM}$ individual primers, and $400 \mathrm{nM}$ dNTP mix (Takara). PCR was conducted by 35 cycles of strand separation at $94^{\circ} \mathrm{C}$ for $60 \mathrm{~s}$, annealing at 50 to $58^{\circ} \mathrm{C}$ based on the Tm values of primers for $60 \mathrm{~s}$, and synthesis at $72^{\circ} \mathrm{C}$ for 1 to 3 min depending on the product size $(1 \mathrm{~min}$ for $1 \mathrm{~kb})$. A final reaction was conducted at $72^{\circ} \mathrm{C}$ for $10 \mathrm{~min}$. The PCR products were analyzed by $1 \%$ agarose gel electrophoresis and then eluted from gels by the Micro-Elute DNA Clean/Extraction kit (GMbiolab) following the standard procedures supplied by the manufacturer. The eluted DNA fragments were ligated to the pCR2.1-TOPO vector using the TOPO TA Cloning kit (Invitrogen) and transferred into E. coli $\mathrm{DH} 5 \alpha$ competent cells according to the manufacturer's instructions. Three clones for each PCR product were selected for sequencing. Nucleotide (nt) sequences of amplicons were determined using the 3730XL DNA Analyzer (Applied Biosystems, Thermo Fisher Scientific, Waltham, MA), performed by Mission Biotech Company (Taipei, Taiwan).

Table 1. Accession codes of the complete genome sequences of different tomato chlorosis virus (ToCV) isolates available in GenBank

\begin{tabular}{lccc}
\hline & & \multicolumn{2}{c}{ Length/accession code } \\
\cline { 3 - 4 } Virus isolate & Country & \multicolumn{1}{c}{ RNA-1 } & RNA-2 \\
\hline AT80/99 & Spain & $8,594 \mathrm{nt} / \mathrm{DQ} 983480$ & $8,244 \mathrm{nt} / \mathrm{DQ} 136146$ \\
Gr-535 & Greece & $8,594 \mathrm{nt} / \mathrm{EU} 284745$ & $8,242 \mathrm{nt} / \mathrm{EU} 284744$ \\
Florida & U.S.A. & $8,595 \mathrm{nt} / \mathrm{AY} 903447$ & $8,247 \mathrm{nt} / \mathrm{AY} 903448$ \\
ToC-Br2 & Brazil & $8,594 \mathrm{nt} / \mathrm{JQ952600}$ & $8,242 \mathrm{nt} / \mathrm{JQ952601}$ \\
SDSG & China & $8,594 \mathrm{nt} / \mathrm{KC709509}$ & $8,242 \mathrm{nt} / \mathrm{KC} 709510$ \\
IS29 & Korea & $8,594 \mathrm{nt} / \mathrm{KP114538}$ & $8,242 \mathrm{nt} / \mathrm{KP114529}$ \\
\hline
\end{tabular}
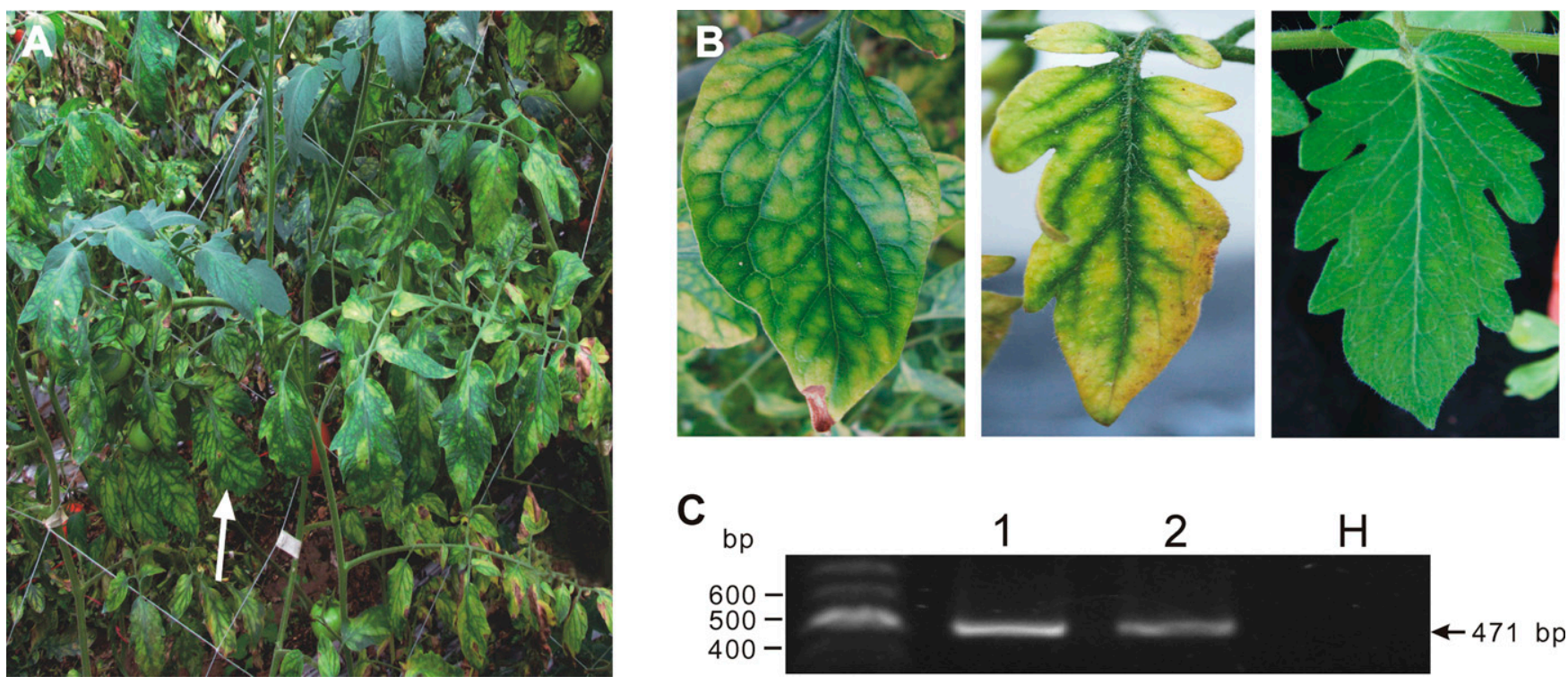

Fig. 1. Isolation of the virus isolate XS from a diseased tomato sample collected from a field in Xinshe District, Taichung, Taiwan. A, chlorosis developed on lower leaves of a tomato plant (indicated by an arrow). B, symptoms on leaves of a naturally infected tomato plant (left) and a XS-whitefly transmitted tomato plant (middle). A healthy tomato leaf is shown for comparison (right). C, virus detection by the nested reverse transcription-polymerase chain reaction method using the degenerate primer sets to viruses of Closteroviridae (Dovas and Katis 2003). Lanes 1 and 2 represent the total RNAs extracted from the field diseased and XS-infected tomato samples, respectively; and lane $H$ represents the total RNA extracted from a healthy tomato leaf as a negative control. The expected size of amplicons is indicated by an arrow. 
The 5' - and 3 '-end sequences of RNA-1 and RNA-2 were verified by rapid amplification of cDNA ends (RACE) following the previous method (Li et al. 2011). Twenty units of SuperScript III reverse transcription (Invitrogen) were used to synthesize first strand cDNAs for tailing. The tailed cDNA fragments were used as templates for the second PCR amplification. The final PCR products were analyzed and sequenced as described above.

Sequence analyses. Comparison of the determined genomic sequences of XS with those of other ToCV isolates and different crinivirus species obtained from GenBank (Table 1 and Supplementary Table S2, respectively) was carried out by the BL2SEQ program of Biology Workbench, SDSC. The nt sequences were translated into aa sequences by the Sixframe program of Biology Workbench, SDSC. In addition, the homologies of nt and aa sequences were calculated by the Gap program of SeqWeb (Accelrys Inc., San Diego, CA). Multiple sequence alignments were performed by the ClustalW program of Biology Workbench, SDSC. Phylogenetic analyses were conducted by the programs of Phylip 3.69 (Department of Genetics, University of Washington, Seattle, WA), using the method of neighbor-joining (Saitou and Nei 1987) with 1,000 bootstrap iterations. Versions of the input data sets were reassembled by the Seqboot program of Phylip 3.69. Distance matrixes of aa sequences were produced by the Protdist program using PAM matrixes of Dayhoff model (Dayhoff et al. 1979).

Virus detection for field samples. Calculated by the PRIMER3 program of Biology Workbench, SDSC, the selected primer pairs XS-R2-847/XS-R2-1526r, XS-R2-3347/XS-R2-3951r, and XS-R24366/XS-R2-5059r, which were designed from the Hsp70h (nt 847 to 1,526 ), P59 (nt 3,347 to 3,951 ), and CP (nt 4,366 to 5,059) ORFs of the ToCV-XS RNA-2, respectively, were used for detecting the
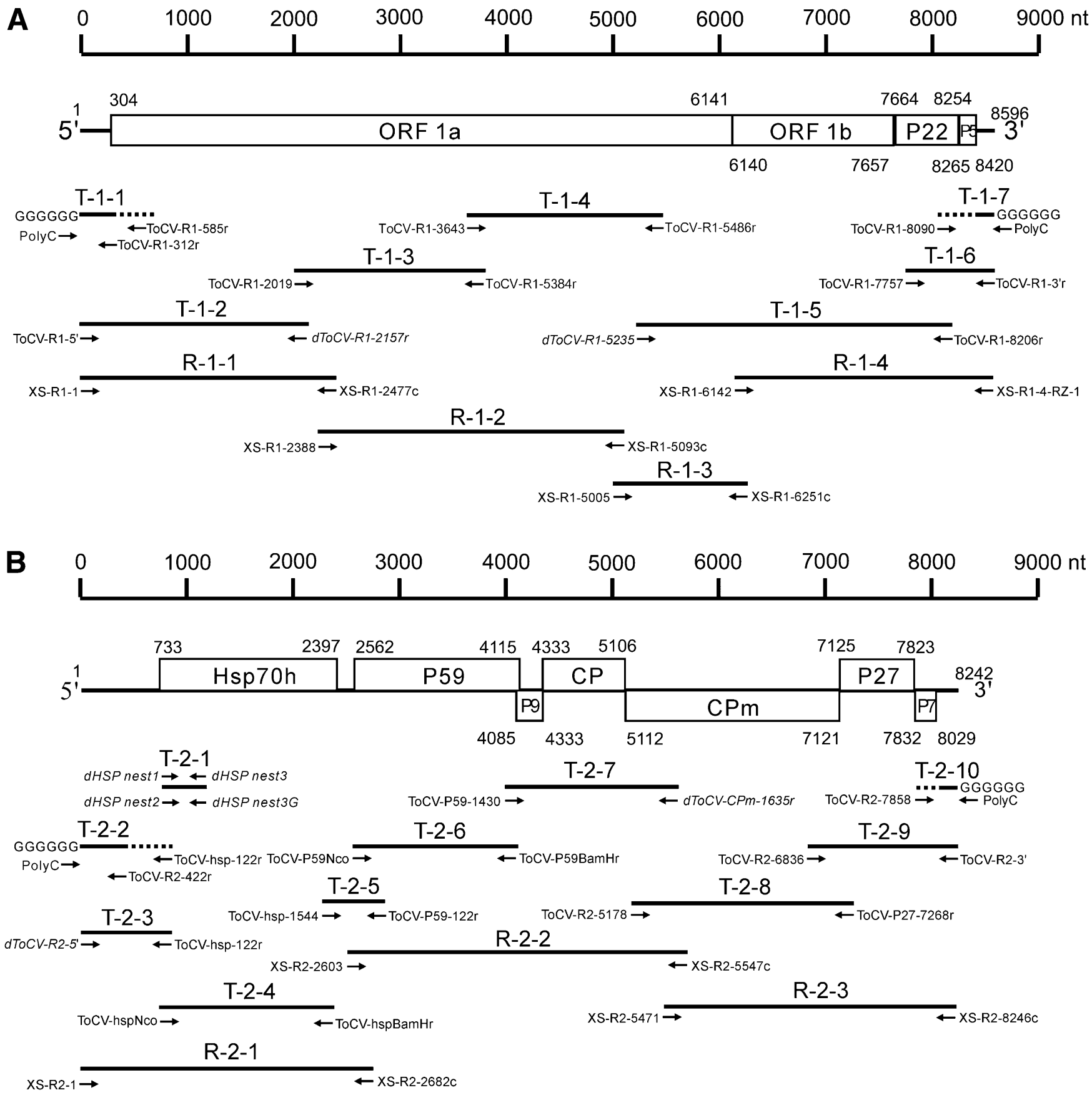

Fig. 2. Strategy for cloning and sequencing of RNA-1 (A) and RNA-2 (B) of the Taiwanese XS isolate of tomato chlorosis virus (ToCV). The open reading frames (ORFs) are represented by boxes, and the nucleotide positions of the individual ORFs on each RNA segment are indicated. The noncoding regions are drawn by lines. Primers used for reverse transcription-polymerase chain reaction amplification and rapid amplification of cDNA ends are indicated by arrows. The degenerate primers were shown in italics. The amplicons are indicated by bold lines. The details of primers are mentioned in Supplementary Table S1. 
XS-related isolates in the collected field samples. A serial dilution of the total RNA extracted from leaves of an XS-infected tomato plant with typical chlorosis symptom was used to evaluate the sensitivity of the designed primer pairs. The total RNAs extracted from ToCVfree tomato and whitefly samples were used for comparison with evaluate the specificity of the primer pairs. A total of 172 symptomatic tomato samples and 177 whiteflies in the diseased tomato fields were collected from cultivated fields in Taoyuan, Taichung, Changhua, Nantou, Yunlin, Chiayi, and Tainan from January 2013 to March 2016 for the detection of ToCV by RT-PCR. The total RNAs of the field tomato samples were extracted using the Plant Total RNA Miniprep Purification kit (GMbiolab) and total RNAs of individual whiteflies collected from diseased tomato fields were extracted by the Total RNA Purification kit (GMbiolab). The total RNA was quantitated by a spectrophotometer (Eppendorf, Hauppauge, NY). RT-PCR was conducted by the One-step RT-PCR kit (GMbiolab) according to the manufacturer's instructions. The reaction mixture consists of $1 \mu \mathrm{g}$ of total RNA, $200 \mathrm{nM}$ individual primers, 25 U One-step RT-PCR enzyme mix, $5 \mu l$ reaction buffer, and $5 \mu l$ enhancer buffer in a $25 \mu l$ volume. The amplification was carried out at $50^{\circ} \mathrm{C}$ for $30 \mathrm{~min}$ for RT, and 35 cycles of strand separation at $94^{\circ} \mathrm{C}$ for $30 \mathrm{~s}$, annealing at $56^{\circ} \mathrm{C}$ for 30 $\mathrm{s}$, extension at $72^{\circ} \mathrm{C}$ for $1 \mathrm{~min}$, and an additional final reaction at $72^{\circ} \mathrm{C}$ for $7 \mathrm{~min}$ for PCR. The PCR products were analyzed by $1 \%$ agarose gel electrophoresis.

\section{Results}

Identification and isolation of ToCV from field samples. A 471-bp DNA fragment was amplified in nested RT-PCR from five tomato samples exhibiting chlorosis (Fig. 1A and B) as shown in Figure 1C (lane 1). One amplicon was directly sequenced and the BLAST result revealed that it shares the highest $82 \%$ nt identity and $98 \%$ aa identity with the partial Hsp70h sequence of ToCVAT80/99 available in GenBank, suggesting that the causal virus for the tomato chlorosis disease is the ToCV.

The field tomato samples with the ToCV sequence were further used as a source for virus isolation by three consecutive passages of whitefly transmission to obtain a putative pure virus culture. The symptoms of chlorosis and green veins, similar to the symptoms of the original diseased plant, were observed on mature leaves of the tomato plants 4 weeks after whitefly inoculation (Fig. 1B). This virus isolate was verified by the nested RT-PCR amplification (Fig. 1C, lane 2) and was designated as XS for further characterization.

Whole genome sequence of XS. The complete nt sequence of XS RNA-1 (accession no. KY618796) was 8,596 nt in length, containing four ORFs of $1 \mathrm{a}, 1 \mathrm{~b}, \mathrm{P} 22$, and P5. The ORF $1 \mathrm{~b}$ is translated via $\mathrm{a}+1$ ribosomal frameshifting of the ORF 1a. The XS RNA-2 (KY618797) is 8,242 nt long, containing seven ORFs coding for Hsp70h, P59, P9, $\mathrm{CP}, \mathrm{CPm}, \mathrm{P} 27$, and $\mathrm{P} 7$. The corresponding nt positions of all ORFs in RNA-1 and RNA-2 of XS are illustrated in Figure 2. The genome organization of XS is distinct from those of the characterized ToCV isolates from Brazil, China, Greece, Korea, Spain, and the United States, reflecting in the results that the $\mathrm{P} 4$ and P8 ORFs before and after the Hsp70h ORF, respectively, are absent in the XS RNA-2 (Supplementary Fig. S1).

Comparison of the genomic sequences of $\mathrm{XS}$ with those of other ToCV isolates and different criniviruses. The complete genome sequence of XS was compared with those of geographically different ToCV isolates. All other ToCV isolates originating from America, Asia, and Europe, are closely related, sharing high levels of 97.3 to $99.5 \%$ nt identity for RNA-1 and 97.6 to $99.3 \%$ nt identity for RNA-2. However, the RNA-1 and RNA-2 of XS share lower levels of 77.8 to $78 \%$ and 78.0 to $78.1 \%$ nt identities, respectively, with

Table 2. Comparison of the complete genome sequence of the XS isolate of tomato chlorosis virus (ToCV) with those of other ToCV isolates from different geographic origins ${ }^{\mathrm{a}}$

\begin{tabular}{|c|c|c|c|c|c|c|c|c|c|c|c|c|c|c|c|c|c|c|c|}
\hline \multirow[b]{2}{*}{ ToCV isolate } & \multirow[b]{2}{*}{ Country } & \multirow[b]{2}{*}{ RNA1 (nt) } & \multicolumn{2}{|c|}{ 1a } & \multicolumn{2}{|c|}{$1 b$} & \multicolumn{2}{|c|}{ P22 } & \multirow[b]{2}{*}{ RNA2 (nt) } & \multicolumn{2}{|c|}{ Hsp70h } & \multicolumn{2}{|c|}{ P59 } & \multicolumn{2}{|c|}{$\mathrm{CP}$} & \multicolumn{2}{|c|}{ CPm } & \multicolumn{2}{|c|}{ P27 } \\
\hline & & & nt & aa & nt & aa & nt & aa & & nt & aa & nt & aa & nt & aa & nt & aa & nt & aa \\
\hline Florida & U.S.A. & 77.8 & 76.8 & 86.8 & 83.5 & 96.2 & 68.9 & 65.2 & 78.0 & 80.4 & 94.4 & 79.3 & 90.9 & 76.6 & 89.4 & 76.6 & 81.1 & 81.8 & 90.0 \\
\hline ToBr2 & Brazil & 78.0 & 76.9 & 87.3 & 83.4 & 96.2 & 69.0 & 65.2 & 78.1 & 80.3 & 94.4 & 79.8 & 91.1 & 76.2 & 88.7 & 76.6 & 81.1 & 82.4 & 90.5 \\
\hline АТ80/99 & Spain & 78.0 & 76.7 & 86.7 & 83.3 & 96.0 & 70.1 & 65.8 & 78.0 & 80.7 & 94.4 & 79.7 & 90.5 & 76.8 & 89.1 & 76.2 & 80.7 & 81.6 & 90.5 \\
\hline SDSG & China & 77.9 & 76.8 & 87.0 & 83.5 & 96.2 & 69.0 & 64.7 & 78.1 & 80.3 & 94.7 & 79.7 & 91.1 & 76.7 & 89.4 & 76.6 & 81.1 & 81.8 & 90.0 \\
\hline Gr535 & Greece & 77.8 & 76.8 & 86.8 & 83.4 & 96.2 & 68.3 & 64.2 & 78.1 & 80.4 & 94.4 & 79.7 & 91.2 & 76.4 & 88.3 & 76.7 & 81.3 & 82.2 & 90.0 \\
\hline IS29 & Korea & 77.9 & 76.9 & 87.4 & 83.4 & 96.2 & 69.2 & 65.2 & 78.1 & 80.3 & 95.1 & 79.4 & 90.9 & 76.6 & 88.7 & 76.6 & 81.0 & 82.2 & 89.6 \\
\hline
\end{tabular}

a The nucleotide (nt) and amino acid (aa) identities are indicated as percentage (\%).

Table 3. Identity percentages of the major open reading frames in the genome of the XS isolate of tomato chlorosis virus (ToCV) compared with those of other crinivirus species

\begin{tabular}{|c|c|c|c|c|c|c|c|c|c|c|c|c|c|c|}
\hline \multirow[b]{2}{*}{ Species $^{\mathbf{a}}$} & \multicolumn{2}{|c|}{$1 a$} & \multicolumn{2}{|c|}{ 1b } & \multicolumn{2}{|c|}{$\begin{array}{c}\mathbf{P} 22 / \mathbf{P}^{2} 3^{\mathrm{b}} / \\
\mathbf{P}^{\mathbf{c}}\end{array}$} & \multicolumn{2}{|c|}{ Hsp70h } & \multicolumn{2}{|c|}{ P59 } & \multicolumn{2}{|c|}{$\mathrm{CP}$} & \multicolumn{2}{|c|}{ CPm } \\
\hline & nt & aa & nt & aa & nt & aa & nt & aa & nt & $\mathbf{a a}$ & nt & aa & nt & aa \\
\hline$\overline{\mathrm{AYV}}$ & $-d$ & - & - & - & - & - & - & - & - & - & 48.1 & 29.9 & - & - \\
\hline BPYV & 51.8 & 36.7 & 63.2 & 62.3 & - & - & 59.9 & 59.6 & 54.2 & 41.0 & 48.2 & 30.2 & 47.3 & 23.9 \\
\hline BYDV & 51.2 & 34.8 & 59.8 & 58.0 & - & - & 61.0 & 61.0 & 53.3 & 42.9 & 47.9 & 30.7 & 36.8 & 21.0 \\
\hline BYVaV & 51.8 & 37.1 & 63.6 & 62.3 & - & - & 60.8 & 59.2 & 52.5 & 39.7 & 49.6 & 28.1 & 47.5 & 23.1 \\
\hline CCYV & 50.9 & 34.4 & 61.3 & 59.5 & 47.0 & 18.5 & 62.3 & 60.8 & 52.8 & 42.6 & 52.5 & 31.0 & 37.1 & 22.4 \\
\hline CYSDV & 50.9 & 34.0 & 62.5 & 61.3 & 43.0 & 14.6 & 62.0 & 61.1 & 54.1 & 40.7 & 51.3 & 34.5 & 34.9 & 21.6 \\
\hline DVCV & 51.7 & 33.8 & 60.8 & 58.5 & - & - & 60.8 & 60.1 & 52.9 & 38.8 & 48.4 & 28.0 & 48.1 & 22.7 \\
\hline LCV & 50.9 & 34.6 & 60.8 & 58.3 & 46.6 & 14.6 & 61.3 & 60.1 & 54.6 & 43.5 & 48.5 & 32.2 & 36.8 & 21.9 \\
\hline LIYV & 51.2 & 29.2 & 56.3 & 53.8 & - & - & 57.3 & 50.2 & 48.7 & 32.1 & 47.6 & 25.8 & 34.6 & 15.5 \\
\hline PYVV & 51.0 & 33.9 & 61.3 & 60.7 & - & - & 61.0 & 58.6 & 52.2 & 36.9 & 48.9 & 34.2 & 47.5 & 22.6 \\
\hline $\mathrm{SPaV}$ & 52.6 & 35.4 & 60.2 & 57.9 & - & - & 62.4 & 61.0 & 51.3 & 40.3 & 48.2 & 26.4 & 46.9 & 23.0 \\
\hline SPCSV & 52.4 & 37.1 & 64.7 & 65.4 & 48.2 & 14.2 & 60.6 & 60.9 & 54.2 & 41.7 & 51.8 & 33.1 & 46.3 & 24.4 \\
\hline TVCV & 51.6 & 35.8 & 64.0 & 60.5 & - & - & 59.4 & 59.4 & 53.7 & 42.9 & 48.5 & 32.2 & 36.4 & 19.3 \\
\hline TICV & 50.1 & 28.9 & 60.4 & 55.5 & - & - & 57.0 & 52.4 & 48.4 & 29.9 & 48.5 & 23.6 & 47.0 & 20.4 \\
\hline
\end{tabular}

${ }^{a}$ See Supplementary Table $\mathrm{S} 2$ for virus abbreviations.

${ }^{b} \mathrm{P} 23$ is the RNA silencing suppressor of LCV.

${ }^{\mathrm{c}} \mathrm{P} 25$ is the RNA silencing suppressor of CYSDV.

d Sequences are unavailable in GenBank. 

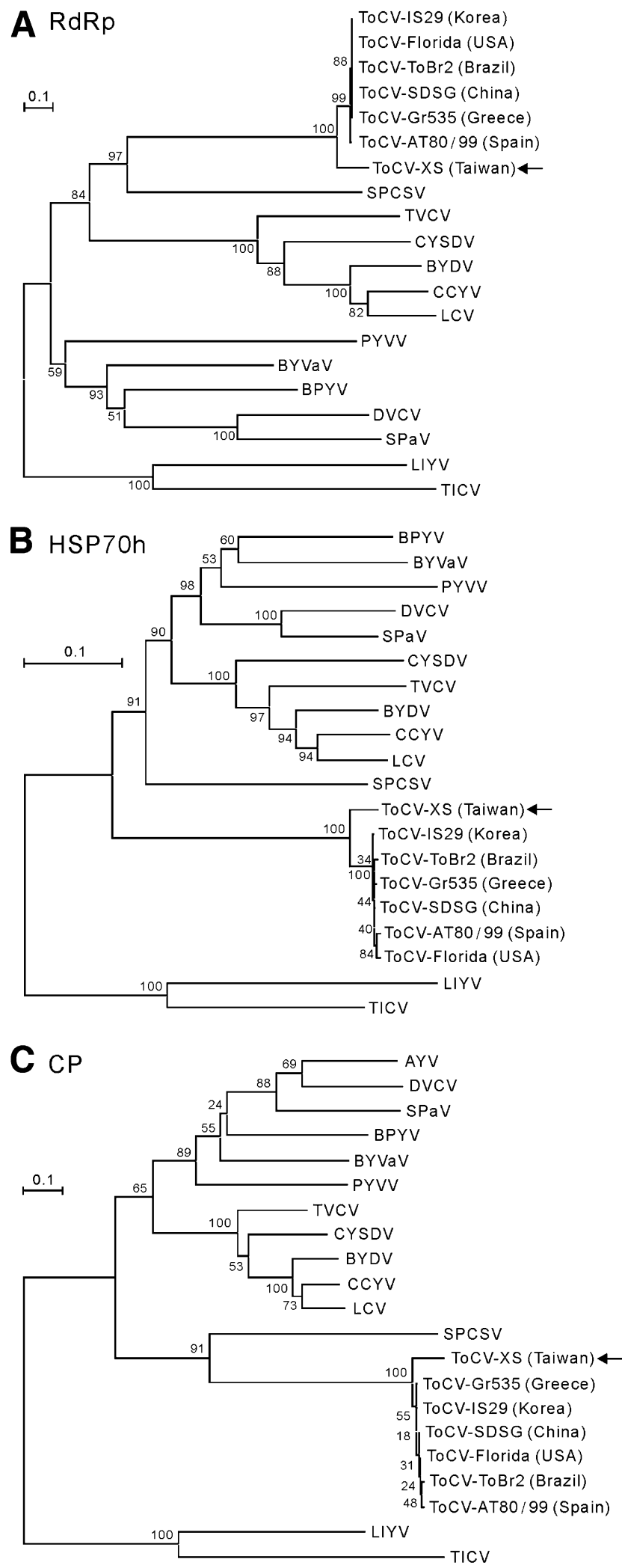

Fig. 3. Phylogenetic analyses of crinivirus species. The three key proteins for classification of criniviruses, including RNA-dependent RNA polymerase (RdRp) (A), heat shock protein 70 homolog (Hsp70h) (B), and major coat protein (CP) (C) were analyzed. The dendrograms were produced using the neighbor-joining algorithm with 1,000 bootstrap replicates (percentages are indicated). The XS isolate of tomato chlorosis virus (ToCV-XS) is indicated by arrows. The details of other ToCV isolates are mentioned in Table 1. The details of different criniviruses are mentioned in Supplementary Table S2. those of different ToCV isolates (Table 2). For RNA-1, the 1a and 1b ORFs of XS share 76.7 to $76.9 \%$ nt identity ( 86.7 to $87.4 \%$ aa identity), and 83.3 to $83.5 \%$ nt identity ( 96 to $96.2 \%$ aa identity) with those of the foreign ToCV isolates, respectively. However, the P22 ORF of XS shares the lowest nt and aa identities of 68.3 to $70.1 \%$ and 64.2 to $65.8 \%$, respectively, with those of other foreign ToCV isolates (Table 2). In RNA-2, the XS Hsp70h ORF shares nt (aa) identities of 80.3 to $80.7 \%$ ( 94.4 to $95.1 \%$ ); P59 ORF shares 79.3 to $79.8 \%$ ( 90.5 to $91.2 \%$ ); CP ORF shares 76.2 to $76.8 \%$ (88.3 to $89.4 \%$ ); CPm ORF shares 76.2 to $76.7 \%$ (80.7 to $81.3 \%$ ); and P27 ORF shares 81.6 to $82.4 \%$ ( 89.6 to $90.5 \%$ ), with those of other known ToCV isolates (Table 2). Our results indicated that XS belonged to the ToCV species based on the threshold of $75 \%$ aa identity of the hallmark proteins RdRp, Hsp70h, and CP, but probably represented a divergent strain of the virus found in Taiwan.

Furthermore, the major ORFs in the genome of XS, including the ORFs 1a, 1b, and P22 in RNA-1 and the ORFs Hsp70h, P59, CP, and $\mathrm{CPm}$ in RNA-2, were compared with those of different crinivirus species, and the results are shown in Table 3. In RNA-1, the 1a and $1 \mathrm{~b}$ ORFs of XS share 50.1 to $52.6 \%$ nt identity (28.9 to $37.1 \%$ aa identity), and 56.3 to $64.7 \%$ nt identity ( 53.8 to $65.4 \%$ aa identity) with those of other criniviruses, respectively. The P22 homologs only present in the RNA-1 of CCYV, CYSDV, LCV, and SPCSV and share 43.0 to $48.2 \%$ nt identity ( 14.2 to $18.5 \%$ aa identity) with the P22 ORF of XS. In RNA-2, the XS Hsp70h ORF shares nt (aa) identities of 57.0 to $62.4 \%$ (50.2 to $61.1 \%$ ); P59 ORF shares 48.4 to $54.6 \%$ (29.9 to $43.5 \%$ ); CP ORF shares 47.6 to $52.5 \%$ (23.6 to $34.5 \%$ ); and CPm ORF shares 34.6 to $48.1 \%$ (15.5 to $24.4 \%$ ), with those of other criniviruses. Our results indicated that XS is a distinct crinivirus species.

The phylogenetic relationships of the key proteins, RdRp, Hsp70h, and $\mathrm{CP}$, were also analyzed. Results indicated that XS is clearly clustered in the ToCV clade (Fig. 3). However, the Taiwanese XS isolate was placed in a distinct branch, separate from ToCV isolates from Brazil, China, Greece, Korea, Spain, and the U.S.A. that shared high levels of identity with each other ( $99.6 \%$ for RdRp, 96.8 to $99.1 \%$ for Hsp70h, and 88.3 to $95.1 \%$ for $\mathrm{CP}$ ).

Primers designed for detection of XS by RT-PCR. The expected cDNA fragments of 680,605 , and $694 \mathrm{bp}$ were specifically amplified from the total RNA extracted from the XS-infected tomato plant by one-step RT-PCR using the corresponding primer pairs of XS-R2-847/XS-R2-1526r, XS-R2-3347/XS-R2-3951r, and XS-R24366/XS-R2-5059r, respectively. Amplifications with the primer pairs XS-R2-3347/XS-R2-3951r and XS-R2-4366/XS-R2-5059r showed higher sensitivity (10 ng) than the primer pair XS-R2-847/ XS-R2-1526r (100 ng) for detecting XS from the total RNA of infected tomato tissues (Fig. 4A). Moreover, the total RNAs extracted from the XS-viruliferous silverleaf whitefly individuals were used as templates for the one-step RT-PCR amplification using the aforementioned primer pairs. Only the primer pair XS-R2-3347/XS-R2$3951 \mathrm{r}$ was able to amplify the expected DNA fragment of $605 \mathrm{bp}$ from a single XS-viruliferous whitefly (Fig. 4B). According to the results of the detection sensitivity and specificity of XS from the tomato and whitefly samples, the primer pair XS-R2-3347/XS-R2-3951r was selected to detect the Taiwanese ToCV isolates in field surveys, and the expected fragment of $605 \mathrm{bp}$ corresponding to the position $\mathrm{nt}$ 3,347 to 3,951 of ToCV-XS RNA-2 was amplified in the tomato and whitefly samples collected from the diseased fields (Fig. 4C).

Detection of the ToCV in field samples. Up to $100 \%$ of diseased tomato plants showing leaf curl can be observed in open fields. Among those diseased tomato plants, 172 tomato samples showing chlorosis on lower leaves and 177 whiteflies in the diseased tomato fields were collected from cultivated fields in Nantou, Taoyuan, Taichung, Tainan, and Yunlin from January 2013 to March 2016 for detecting ToCV-XS by RT-PCR using the primer pair XS-R23347/XS-R2-3951r. The result showed that 104 tomato samples $(60.5 \%)$ and 51 individual whiteflies $(28.8 \%)$ were ToCV-XS positive (Table 4). The ToCV-XS can be detected in most of areas, including northern (Taoyuan), central (Taichung, Changhua, Nantou, and Yunlin), and southern (Chiayi and Tainan) regions of Taiwan 
(Table 4), indicating that the XS strain of ToCV is prevalent in Taiwan. Only a few of amplicons were sequenced and exhibited near $100 \%$ nt identity to the sequence of ToCV-XS.

\section{Discussion}

The occurrence of ToCV in Taiwan in 1998 was identified by RNA hybridization using a ToCV-CP probe (Tsai et al. 2004), but the genomic sequence of the virus was not determined. In this study, the presence of ToCV in Taiwan was revalidated by the nested RTPCR method using Hsp70h-specific degenerate primers (Dovas and Katis 2003). Furthermore, the entire genome sequence of a Taiwanese isolate XS was determined. Although the ToCV-XS genome only shares about $78 \%$ nt identity with other geographically different ToCV isolates, its individual ORFs share a high aa identity (80.7 to $96.2 \%$ ), except for P22 (64.2 to 65.8\%). The identity of XS as an isolate from the ToCV species is validated based on the criteria for the classification of a Crinivirus species with a threshold of $75 \%$ aa identity in the RdRp, Hsp70h, and CP set by the International Committee on Taxonomy of Viruses (Martelli et al. 2011). On the other hand, the sequences of RdRp, Hsp70h, and $\mathrm{CP}$ of ToCV that are available in GenBank were also analyzed using BLAST. Most of them share similar identities with ToCV-XS as the results shown in Table 2 except the Hsp70h of the GD01 isolate (accession no. KT962275), which was collected from Guangdong, China, shares the highest nt and aa identities of $97 \%$ with that of the Taiwanese XS isolate. We concluded that the GD01 isolate may also belong to the same ToCV-XS strain.

Our field survey result showed that using the XS-specific primers the ToCV can be detected in most of areas, including northern (Taoyuan), central (Taichung, Changhua, Nantou, and Yunlin), and southern (Chiayi and Tainan) regions of Taiwan (Table 4), indicating that the XS-type isolates of ToCV is prevalent in Taiwan. Only a few of amplicons were sequenced and exhibited near 100\% nt identity to the sequence of ToCV-XS.

RdRp, Hsp70h, and CP, sharing a high homology (88.3 to $96.2 \%$ of aa identities) with other geographically different ToCV isolates, are hallmark genes in the family Closteroviridae (Martelli et al. 2011). Actually, these three proteins are essential for the infection of criniviruses in host plants. The criniviral RdRp is responsible for virus replication and the $\mathrm{CP}$ is responsible for virion structure. $\mathrm{Hsp} 70 \mathrm{~h}$ is also associated with genome replication and subgenomic RNA synthesis and virion assembly (Klaassen et al. 1995; Peremyslov
A

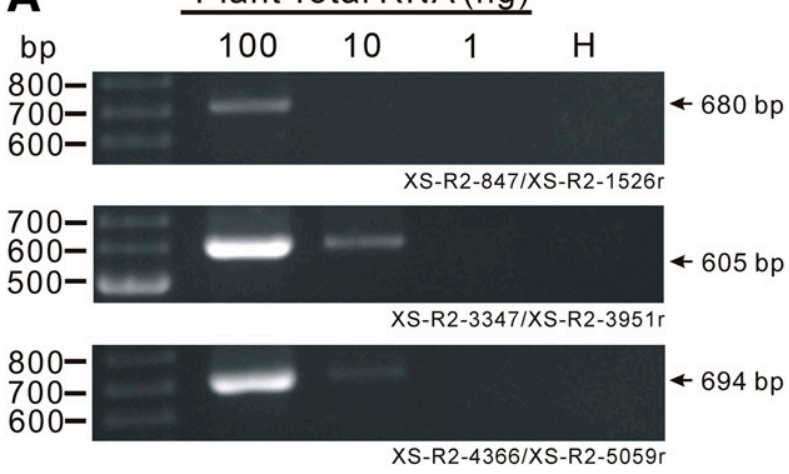

B
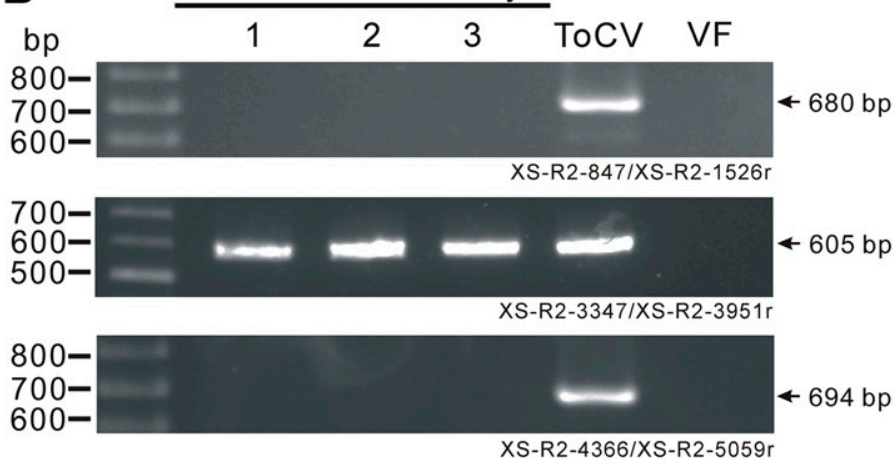

XS-R2-4366/XS-R2-5059r

C

Tomato plant Viruliferous whitefly

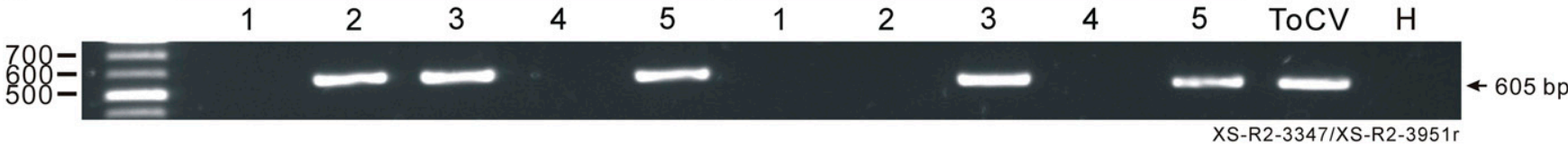

Fig. 4. Detection of tomato chlorosis virus (ToCV) by the one-step reverse transcription-polymerase chain reaction using the primer pairs XS-R2-847/XS-R2-1526r (A and $B$ upper panels), XS-R2-3347/XS-R2-3951r (A and B middle panels and C), and XS-R2-4366/XS-R2-5059r (A and B lower panels), specific to the XS isolate of ToCV (ToCV-XS). A, sensitivity assay against an infected tomato plant. Serially diluted total RNA extracted from the ToCV-XS-infected tomato leaves was used for the test. The amounts of total RNAs were quantitated by a spectrophotometer. B, detection of ToCV in individuals of the silverleaf whitefly. Lanes 1 to 3 represent the total RNA extracted from the individual ToCV-XS-acquired silverleaf whiteflies. The total RNA of a ToCV-XS-infected tomato leaf was used as a positive control. The total RNA extracted from a virus-free silverleaf whitefly (VF) was used as a negative control. C, field survey of the ToCV in tomato plants and silverleaf whiteflies, using the primer pair XS-R2-3347/XS-R2-3951r The total RNA extracted from a ToCV-XS-infected tomato plant was used as the positive control and a healthy $(\mathrm{H})$ tomato leaf was used as the negative control. The expected sizes of the individual amplicons are indicated.

Table 4. Field survey of the tomato chlorosis virus XS strain (ToCV-XS) present in diseased tomato plants and whiteflies collected around Taiwan during January 2013 to March 2016

\begin{tabular}{|c|c|c|c|c|c|}
\hline \multicolumn{2}{|c|}{ Location } & \multirow{2}{*}{$\begin{array}{c}\text { Tested tomato no. } \\
9\end{array}$} & \multirow{2}{*}{$\begin{array}{c}\text { ToCV-XS-infected tomato no. } \\
3\end{array}$} & \multirow{2}{*}{$\begin{array}{c}\text { Tested whitefly no. } \\
-\end{array}$} & \multirow{2}{*}{$\begin{array}{c}\text { ToCV-XS-viruliferous whitefly no. } \\
-\end{array}$} \\
\hline Taoyuan & Bade & & & & \\
\hline \multirow[t]{2}{*}{ Taichung } & Wufeng & 20 & 13 & 25 & 19 \\
\hline & Xinshe & 81 & 50 & 40 & 6 \\
\hline Changhua & Xizhou & 16 & 11 & 40 & 20 \\
\hline \multirow[t]{3}{*}{ Nantou } & Zhushan & 4 & 0 & - & - \\
\hline & Zhongxing village & 3 & 2 & 36 & 6 \\
\hline & Puli & 10 & 7 & - & - \\
\hline \multirow[t]{2}{*}{ Yunlin } & Lunbei & 8 & 8 & - & - \\
\hline & Xiluo & 4 & 3 & - & - \\
\hline Chiayi & Taibao & 10 & 1 & 36 & 0 \\
\hline Tainan & Shanhua & 7 & 6 & - & - \\
\hline \multicolumn{2}{|c|}{ Total sample no. } & 172 & $104(60.5 \%)$ & 177 & $51(28.8 \%)$ \\
\hline
\end{tabular}


et al. 1999; Satyanarayana et al. 2000; Tian et al. 1999). Remarkably, the RNA-1-encoded P22 of the ToCV-XS shares the lowest level of homology with those of other geographically different ToCV isolates. The P22 of the ToCV has been reported to act as an effective suppressor of RNA silencing, which is an antiviral defense in planta, with the binding activity to single-stranded (ss) and double-stranded RNA molecules (Cañizares et al. 2008; Landeo-Ríos et al. 2016). The ToCV-XS P22 shares a similar protein structure of putative zinc finger domain in its $\mathrm{N}$-terminal part with those of other ToCV isolates (data not shown), and may act as an RSS of the ToCV-XS. The RNA2encoded CP and CPm of the ToCV were also reported as RSSs (Cañizares et al. 2008). The ToCV CP interacts with S-adenosyl-homocysteine hydrolase $(\mathrm{SAHH})$, a host protein for sustaining the methyl cycle and Sadenosylmethionine-dependent methyltransferase activity, to suppress local silencing (Cañizares et al. 2013). The possible role of the ToCV $\mathrm{CPm}$ involving in RNA silencing suppression remains unknown. The RSS activity of the CP, CPm, and P22 of the ToCV-XS will be further investigated.

On the other hand, the P4 and P8 ORFs are commonly present within the RNA-2 of all other geographically different ToCV isolates in GenBank but are absent in the RNA-2 of the ToCV-XS. The interspecies variation of the ORF number in the ToCV genome is first found in this study. Viruses of the family Closteroviridae have a frequent phenomenon of recombinant-mediated gene gain or loss (Dolja et al. 2006; Karasev 2000; Rubio et al. 2013). The interspecies diversity of the ORF number in genomic RNA segments was also found in other criniviruses. For instance, the P22 of the sweet potato chlorotic stunt virus (SPCSV), acting as viral pathogenicity enhancer and RSS, was found in the Uganda (Ug) isolate but not in other SPCSV isolates from Peru (Cuellar et al. 2008, 2011). This evolutionary phenomenon indicated that the P22 of SPCSV-Ug might be acquired from unknown organisms such as diversified varieties of crops (breeding for resistance) or virus population (mixed infection pressure) (Cuellar et al. 2011; Tugume et al. 2013). Taiwan is located between tropical and subtropical regions, and the World Vegetable Center, located at southern Taiwan, possesses the largest collection of tomato germplasm in the world. Numerous tomato varieties have been released from the center, and the diversified varieties of tomatoes may provide different selection pressure for genetic variability of the ToCV in Taiwan. Nevertheless, the significance of a very different P22 and the diversity of ORF numbers between ToCV-XS and other foreign isolates remains to be further investigated.

The field survey of criniviruses is mostly conducted by nucleic acid assay, such as RT-PCR (Varanda et al. 2015) and RT-loopmediated isothermal amplification assay (Zhao et al. 2015), due to the low titer of phloem-limited criniviruses in infected plant tissues. The completion of whole genome sequencing of the ToCV-XS greatly helps to design efficient primers for identification and detection of the ToCV isolates in Taiwan. Primers designed from the CP and Hsp70h genes are frequently used for RT-PCR amplification (Hirota et al. 2010; Louro et al. 2000; Navas-Castillo et al. 2000; Segev et al. 2004). In our case, the primer pair XS-R2-3347/XSR2-3951r, designed from the P59 gene, has a higher sensitivity than the primer pairs XS-R2-847/XS-R2-1526r from the Hsp70h gene and XS-R2-4366/XS-R2-5059r from the CP gene for detection of ToCV in infected tomato plants and in a single viruliferous silverleaf whitefly. The primer pairs used in this study are ToCV-XS-specific and used to detect ToCV-XS-related isolates. Actually, we also attempted to develop a ToCV-universal detection method for detecting all ToCV isolates, and it can be successfully used to detect ToCV-XS (data not shown). No ToCV isolates that are different from ToCVXS have been found in Taiwan so far. The disease prevalence of the ToCV-XS-related isolates in Taiwan was thus monitored by RT-PCR in this study and our results revealed that ToCV has become an emerging threat for tomato production.

In the past decade, global warming led to an increase of the whitefly populations. The high incidence of ToCV in Taiwan may be a result from the warmer winters in recent years, which have contributed to the increase of propagation and spread of whiteflies $B$. tabaci B biotype. Whiteflies $B$. tabaci B biotype are also the transmission vector of several important tomato-infecting begomoviruses, including ageratum yellow vein Hualian virus, tomato leaf curl Hsinchu virus, tomato leaf curl Taiwan virus, and tomato yellow leaf curl Thailand virus (TYLCTHV) in Taiwan (Tsai et al. 2011). Occurrence of ToCV epidemics in many countries has become a major concern for tomato production, not only because of its direct damage but also because of synergistic effects with other viruses. The high incidence of diseased tomato samples of ToCV implies a possible mixed infection with other viruses, especially begomoviruses, because they share the same whitefly vector. TYLCTHV is currently rampant throughout the entire island of Taiwan (Jan et al. 2007), and the typical leaf curl symptom is commonly observed and up to $100 \%$ infection rate of TYLCTHV could be detected when field surveys were conducted (data not shown). It cannot exclude the possibility that the chlorosis syndrome observed on the other ToCV-negative tomato samples was caused by physiological disorder or infections with other viruses. This complexity complies with the recently emerged thrips-borne tomato spotted wilt virus (TSWV) in Taiwan (Zheng et al. 2010), which will make the production of tomatoes become increasingly difficult.

Integrated pest management (IPM) by spreading insecticides and removal of weeds to reduce insect vector populations is the common measure for control of viral diseases. An approach to concurrently provide resistance to an ssDNA virus and an ssRNA virus has been developed in our laboratory (Yang et al. 2014). This approach is being adapted to generate the concurrent transgenic resistance to whiteflyborne geminiviruses and criniviruses, and thrips-borne tospoviruses in tomato and other cucurbit crops.

In conclusion, the ToCV prevailing in Taiwan represents a new strain, named ToCV-XS, and it has become a serious threat for the production of tomatoes in Taiwan. The molecular characterization of ToCV-XS and the development of the RT-PCR-based detection tool will help to understand the dynamics of tomato chlorosis disease epidemics, and also help to develop feasible control measures, either by conventional breeding or transgenic resistance.

\section{Acknowledgments}

Our gratitude goes to Michael Burton, Asia University.

\section{Literature Cited}

Al Rwahnih, M., Dolja, V. V., Daubert, S., Koonin, E. V., and Rowhani, A. 2012 Genomic biological analysis of Grapevine leafroll-associated virus 7 reveals a possible new genus within the family Closteroviridae. Virus Res. 163:302-309.

Al-Saleh, M. A., Al-Shahwan, I. M., Shakeel, M. T., Amer, M. A., Orfanidou, C. G., and Katis, N. I. 2014. First report of Tomato chlorosis virus (ToCV) in tomato crops in Saudi Arabia. Plant Dis. 98:1590.

Cañizares, M. C., Lozano-Duran, R., Canto, T., Bejarano, E. R., Bisaro, D. M., Navas-Castillo, J., and Moriones, E. 2013. Effects of the crinivirus coat protein-interacting plant protein SAHH on post-transcriptional RNA silencing and its suppression. Mol. Plant-Microbe Interact. 26:1004-1015.

Cañizares, M. C., Navas-Castillo, J., and Moriones, E. 2008. Multiple suppressors of RNA silencing encoded by both genomic RNAs of the crinivirus, Tomato chlorosis virus. Virology 379:168-174.

Cuellar, W. J., Cruzado, K. R., Fuentes, S., Untiveros, M., Soto, M., and Kreuze, J. F. 2011. Sequence characterization of a Peruvian isolate of Sweet potato chlorotic stunt virus: further variability and a model for p22 acquisition. Virus Res. 157:111-115.

Cuellar, W. J., Tairo, F., Kreuze, J. F., and Valkonen, J. P. T. 2008. Analysis of gene content in Sweet potato chlorotic stunt virus RNA1 reveals the presence of p22 RNA silencing suppressor in only few isolates: implications to viral evolution and synergism. J. Gen. Virol. 89:573-582.

Dayhoff, M. O., Schwartz, R. M., and Orcutt, B. C. 1979. A model of evolutionary change in protein. Pages 345-352 in: Atlas of Protein Sequence and Structure. M. O. Dayhoff, ed. National Biomedical Research Foundation, Washington, D.C.

Dolja, V. V., Kreuze, J. F., and Valkonen, J. P. T. 2006. Comparative and functional genomics of closteroviruses. Virus Res. 117:38-51.

Dovas, C. I., and Katis, N. I. 2003. A spot multiplex nested RT-PCR for the simultaneous and generic detection of viruses involved in the aetiology of grapevine leafroll and rugose wood of grapevine. J. Virol. Methods 109: 217-226

Dovas, C. I., Katis, N. I., and Avgelis, A. D. 2002. Multiple detection of criniviruses associated with epidemics of a yellowing disease of tomato in Greece. Plant Dis. 86:1345-1349.

Fiallo-Olivé, E., Hamed, A. A., Moriones, E., and Navas-Castillo, J. 2011. First report of Tomato chlorosis virus infecting tomato in Sudan. Plant Dis. 95:1592.

Hirota, T., Natsuaki, T., Murai, T., Nishigawa, H., Niibori, K., Goto, K., Hartono, S., Suastika, G., and Okuda, S. 2010. Yellowing disease of tomato caused by 
Tomato chlorosis virus newly recognized in Japan. J. Gen. Plant Pathol. 76: 168-171.

Jan, F. J., Green, S. K., Shih, S. L., Lee, L. M., Ito, H., Kimbara, J., Hosoi, K., and Tsai, W. S. 2007. First report of Tomato yellow leaf curl Thailand virus in Taiwan. Plant Dis. 91:1363.

Jelkmann, W., Mikona, C., Turturo, C., Navarro, B., Rott, M. E., Menzel, W., Saldarelli, P., Minafra, A., and Martelli, G. P. 2012. Molecular characterization and taxonomy of grapevine leafroll-associated virus 7. Arch. Virol. 157: 359-362.

Karasev, A. V. 2000. Genetic diversity and evolution of closteroviruses. Annu. Rev. Phytopathol. 38:293-324.

Kataya, A. R., Suliman, M. N., Kalantidis, K., and Livieratos, I. C. 2009. Cucurbit yellow stunting disorder virus p25 is a suppressor of post-transcriptional gene silencing. Virus Res. 145:48-53.

Klaassen, V. A., Boeshore, M. L., Koonin, E. V., Tian, T., and Falk, B. W. 1995. Genome structure and phylogenetic analysis of lettuce infectious yellows virus, a whitefly-transmitted, bipartite closterovirus. Virology 208:99-110.

Landeo-Ríos, Y., Navas-Castillo, J., Moriones, E., and Cañizares, M. C. 2016. The p22 RNA silencing suppressor of the crinivirus Tomato chlorosis virus preferentially binds long dsRNAs preventing them from cleavage. Virology 488:129-136.

Li, J. T., Yeh, Y. C., Yeh, S. D., Raja, J. A., Rajagopalan, P. A., Liu, L. Y., and Chen, T. C. 2011. Complete genomic sequence of watermelon bud necrosis virus. Arch. Virol. 156:359-362.

Louro, D., Accotto, G. P., and Vaira, A. M. 2000. Occurrence and diagnosis of Tomato chlorosis virus in Portugal. Eur. J. Plant Pathol. 106:589-592.

Martelli, G. P., Abou Ghanem-Sabanadzovic, N., Agranovsky, A. A., Al Rwahnih, M., Dolja, V. V., Dovas, C. I., Fuchs, M., Gugerli, P., Hu, J. S., Jelkmann, W., Katis, N. I., Maliogka, V. I., Melzer, M. J., Menzel, W., Minafra, A., Rott, M. E., Rowhani, A., Sabanadzovic, S., and Saldarelli, P. 2012. Taxonomic revision of the family Closteroviridae with special reference to the grapevine leafroll-associated members of the genus Ampelovirus and the putative species unassigned to the family. J. Plant Pathol. 94:7-19.

Martelli, G. P., Agranovsky, A. A., Bar-Joseph, M., Boscia, D., Candresse, T., Coutts, R. H. A., Dolja, V. V., Hu, J. S., Jelkmann, W., Karasev, A. V., Martin, R. R., Minafra, A., Namba, S., and Vetten, H. J. 2011. Family Closteroviridae. Pages 987-1001 in: Virus Taxonomy. Ninth Report of the International Committee on Taxonomy of Viruses. A. King, M. J. Adams, E. B. Carstens, and E. Lefkowitz, eds. Elsevier-Academic Press, Amsterdam, The Netherlands.

Moodley, V., Gubba, A., and Mafongoya, P. L. 2016. Occurrence of Tomato chlorosis virus (ToCV) on Datura stramonium near tomato crops (Solanum lycopersicum) in South Africa. Plant Dis. 100:1512.

Navas-Castillo, J., Camero, R., Bueno, M., and Moriones, E. 2000. Severe yellowing outbreaks in tomato in Spain associated with infections of Tomato chlorosis virus. Plant Dis. 84:835-837.
Navas-Castillo, J., Fiallo-Olivé, E., and Sánchez-Campos, S. 2011. Emerging virus disease transmitted by whiteflies. Annu. Rev. Phytopathol. 49:219-248.

Peremyslov, V. V., Hagiwara, Y., and Dolja, V. V. 1999. HSP70 homolog functions in cell-to-cell movement of a plant virus. Proc. Natl. Acad. Sci. USA 96:14771-14776.

Rubio, L., Guerri, J., and Moreno, P. 2013. Genetic variability and evolutionary dynamics of viruses of the family Closteroviridae. Front. Microbiol. 4:151.

Saitou, N., and Nei, M. 1987. The neighbor-joining method: a new method for reconstructing phylogenetic trees. Mol. Biol. Evol. 4:406-425.

Satyanarayana, T., Gowda, S., Mawassi, M., Albiach-Martí, M. R., Ayllón, M. A., Robertson, C., Garnsey, S. M., and Dawson, W. O. 2000. Closterovirus encoded HSP70 homolog and p61 in addition to both coat proteins function in efficient virion assembly. Virology 278:253-265.

Segev, L., Wintermantel, W. M., Polston, J. E., and Lapidot, M. 2004. First report of Tomato chlorosis virus in Israel. Plant Dis. 88:1160.

Tian, T., Rubio, L., Yeh, H. H., Crawford, B., and Falk, B. W. 1999. Lettuce infectious yellows virus: in vitro acquisition analysis using partially purified virions and the whitefly Bemisia tabaci. J. Gen. Virol. 80:1111-1117.

Tsai, W. S., Shih, S. L., Green, S. K., Hanson, P., and Liu, H. Y. 2004. First report of the occurrence of Tomato chlorosis virus and Tomato infectious chlorosis virus in Taiwan. Plant Dis. 88:311.

Tsai, W. S., Shih, S. L., Kenyon, L., Green, S. K., and Jan, F. J. 2011. Temporal distribution and pathogenicity of the predominant tomato-infecting begomoviruses in Taiwan. Plant Pathol. 60:787-799.

Tugume, A. K., Amayo, R., Weinheimer, I., Mukasa, S. B., Rubaihayo, P. P., and Valkonen, J. P. T. 2013. Genetic variability and evolutionary implications of RNA silencing suppressor gene in RNA1 of Sweet potato chlorotic stunt virus isolates infecting sweetpotato and related wild species. PLoS One 8:e81479.

Varanda, C. M., Santos, S. J., Oliveira, M. D., Clara, M. I., and Félix, M. R. 2015. Detection of sweet potato virus $\mathrm{C}$, sweet potato virus 2 and sweet potato feathery mottle virus in Portugal. Acta Virol. 59:185-188.

Wintermantel, W. M., and Wisler, G. C. 2006. Vector specificity, host range, and genetic diversity of Tomato chlorosis virus. Plant Dis. 90:814-819.

Wisler, G. C., Li, R. H., Liu, H. Y., Lowry, D. S., and Duffus, J. E. 1998. Tomato chlorosis virus: A new whitefly-transmitted, phloem-limited, bipartite closterovirus of tomato. Phytopathology 88:402-409.

Yang, C. F., Chen, K. C., Cheng, Y. H., Raja, J. A., Huang, Y. L., Chien, W. C., and Yeh, S. D. 2014. Generation of marker-free transgenic plants concurrently resistant to a DNA geminivirus and a RNA tospovirus. Sci. Rep. 4:5717.

Zhao, L. M., Li, G., Gao, Y., Zhu, Y. R., Liu, J., and Zhu, X. P. 2015. Reverse transcription loop-mediated isothermal amplification assay for detecting tomato chlorosis virus. J. Virol. Methods 213:93-97.

Zhao, R. N., Wang, R., Wang, N., Fan, Z. F., Zhou, T., Shi, Y. C., and Chai, M. 2013. First report to Tomato chlorosis virus in China. Plant Dis. 97:1123.

Zheng, Y. X., Huang, C. H., Cheng, Y. H., Kuo, F. Y., and Jan, F. J. 2010. First report of Tomato spotted wilt virus in sweet pepper in Taiwan. Plant Dis. 94:920. 